\title{
Correlation between Intracerebral Hemorrhage Score and surgical outcome of spontaneous intracerebral hemorrhage
}

\author{
Rashid HU, Amin R, Rahman A, Islam MR, Hossain M, Barua KK, Hossain MA \\ Department of Neurosurgery, Bangabandhu Sheikh Mujib Medical University (BSMMU), Dhaka. \\ Email:liton35th@yahoo.com
}

\begin{abstract}
Spontaneous intracerebral hemorrhage (ICH) comprises $10-15 \%$ of all strokes and has a higher risk of morbidity and mortality (40-45\%). A simple and widely valid clinical grading scale, the Intracerebral Hemorrhage Score (ICH score) was developed to predict to outcome of spontaneous ICH. The aim of the present study was to assess the relation between the ICH score and the surgical outcome of ICH by Glasgow Outcome Scale (GOS) at the $30^{\text {th }}$ post ictus day in our perspective. This prospective study was done during the period of April 2009 to October 2010 in Department of Neurosurgery, Bangabandhu Sheikh Mujib Medical University (BSMMU), Dhaka. Forty three cases were enrolled by set inclusion and exclusion criteria. Intracerebral Hemorrhage Score was calculated during admission and the surgical outcome of ICH was determined by GOS by face to face or telephone interview using structured questionnaire on their $30^{\text {th }}$ post ictus day. Correlation between the ICH score and the surgical outcome of ICH was done by Pearson's correlation coefficient test. Value of $r$ was found to be -0.635 which was statistically highly significant $(\mathrm{p}=.001)$ and the relation was found to be negative. Higher ICH score had unfavorable outcome As correlation between the ICH score and the surgical outcome of ICH was found statistically highly significant, it can be used widely as a grading scale in preoperative counseling. The use of ICH score could improve standardization of clinical treatment protocols and clinical research studies in ICH.
\end{abstract}

\section{Introduction}

Spontaneous intracerebral hemorrhage (ICH) is defined as bleeding into brain parenchyma without accompanying trauma. It occurs with an incidence estimated to range from 15 to 35 cases per 100,000 people per year ${ }^{\mathbf{1}}$. Despite improvements in neurological intensive care, less than one-third of patients with ICH make a good functional recovery. Within 30 days of the ICH ictus, 35-52\% of patients are likely to die, and only $20 \%$ are expected to be functionally independent at 6 months. Worst of all, there is no proven effective treatment and tools for predicting the outcome of this fatal disease ${ }^{2}$. Clinical grading scales play an important role in the evaluation and management of patients with acute neurological disorders. Examples of widely used clinical grading scales are the Glasgow Coma Scale (GCS) for traumatic brain injury, the National Institutes of Health Stroke Scale (NIHSS) for ischemic stroke and the Spetzler-Martin scale for Arteriovenous malformation $(\mathrm{AVM})^{\mathbf{3 , 4}}$. However, despite the common occurrence and high morbidity of $\mathrm{ICH}$, there remains no widely used clinical grading scale for ICH. To be generally applicable, a clinical grading scale must be simple enough to use without significant special training, statistical knowledge or extensive time commitment. The ICH score, which is simple and effective, now seems to be a standard neurological assessment tool that is reproducible and reliable for predicting outcome of $\mathrm{ICH}^{5}$.

Because of high mortality and morbidity proper preoperative counseling is very crucial regarding patient management. To do so the ICH score was devised using the 5 variables - GCS, volume of hematoma, age, location of hematoma and intraventricular extension to manage the ICH patients in a better way in the light of proper prediction. The ICH Score was demonstrated accurate in the population from which it was developed. ${ }^{5}$ However, in order for a scale such as the ICH Score to be useful, it must demonstrate validity in other patient populations. The ICH score, which has been shown to have wide validity in different populations, can predict the outcome of $\mathrm{ICH}$ in terms of mortality as well as in terms of functional outcome and disability also. There was no study carried out in our country to see the postoperative functional outcome in respect to $\mathrm{ICH}$ score. The aim of the study was to see the 
relationship between $\mathrm{ICH}$ score and surgical outcome of ICH in terms of disability and mortality in our context. Our study may be able to provide a better understanding of the ICH score and its relationship prediction of clinical outcomes, so that rational therapies can be applied with a successful outcome.

\section{Materials and Methods}

This cohort study was carried out in the Department of Neurosurgery, Bangabandhu Sheikh Mujib Medical University (BSMMU) from April 2009 to October 2010. A total of 43 cases were selected by non-probability purposive sampling according to following criteria.

Inclusion criteria: Patients who had features suggesting of spontaneous intracerebral hemorrhage, patients who had computed tomography (CT scan) evidence of intracerebral hemorrhage and patients who underwent craniotomy / craniectomy for evacuation of spontaneous intracerebral hemorrhage.

Exclusion criteria: Patients who needed artificial maintenance of respiration, patients who underwent burr hole and aspiration or only external ventricular drainage and patients who had co-morbid disease except hypertension and diabetes.

Data regarding history and physical examination was collected from the history sheet. Findings of the CT scan were recorded. ICH score was made for each patient.

Table I: Criteria employed for defining the Intracerebral Hemorrhage score (ICH score)

\begin{tabular}{lc}
\hline \multicolumn{1}{c}{ Component } & ICH score points \\
\hline Glasgow Coma Scale ( GCS ) & 2 \\
$3-4$ & 1 \\
$5-12$ & 0 \\
$13-15$ & \\
Intracerebral hemorrhage volume, $\mathrm{cm}^{3}$ & 1 \\
$\geq 30$ & 0 \\
$<30$ & 1 \\
Intraventricular Hemorrhage (IVH) & 0 \\
Yes & \\
No & 1 \\
Infratentorial origin of intracerebral hemorrha! & 0 \\
Yes & 1 \\
No & 0 \\
Age, years & $0-6$ \\
$\geq 80$ & \\
$<80$ & \\
\hline Total intracerebral hemorrhage score & \\
\hline
\end{tabular}

Patients of intracerebral hemorrhage were divided into two groups: lower risk group \& higher risk group. Patient of lower risk group had $\mathrm{ICH}$ score 0 and 1 and higher risk group had ICH score 2, 3, 4, 5 , 6. Post-operative status of the patient at regular follow ups were recorded in the data collection sheet. Data regarding the age, GCS on admission, volume of the hematoma, presence of hemorrhage in relation to tentorium, presence of intraventricular extension and surgical outcome of the patient was recorded during their hospital stay or using structured questionnaire interview by the GOS for the discharged patients over telephone in their $30^{\text {th }}$ post ictus day.

In this study the term surgical outcome was used to designate the outcome at $30^{\text {th }}$ post ictus day by GOS as:

Score-5: Good recovery, resumption of normal life despite minor deficit ('Return to work' not reliable)

Score-4: Moderate disability (Disable but independent)-Travel by public transportation, can work in sheltered setting (exceeds mere ability to perform "Activity of daily living")

Score-3: Severe disability (Conscious but disabled)-Dependent for daily support.

Score-2: Persistent vegetative stateUnresponsive and speechless; after 2-3 weeks. May open eye and have sleep/ wake cycles.

Score-1: Death.

Outcome was favorable when the patient had the Score 5 and 4 and outcome was unfavorable if the patient had the score $3,2 \& 1$.

Data Analysis: Collected data of each patient was verified and entered into a statistical package for social science (SPSS), $12^{\text {th }}$ edition for analysis. The probability of association between risk group and outcome was analyzed by Fisher's exact test. Statistical calculation (Pearson's correlation coefficient test) was performed to see the relationship between Intracerebral Hemorrhage Score and surgical outcome of intracerebral hemorrhage if there is any. In this study level of significance was $5 \%$ and power of test was $95 \%$.

Data presentation: Data and result is presented in the form of table where applicable.

\section{Result \\ Pre-operative status \\ Age and sex}

In this study among the 43 patient ages ranged from 28 years to 82 years. The peak age incidence of ICH was found in 50-59 years age group and 34 $(79.07 \%)$ were male and $9(20.93 \%)$ were female. The male to female ratio was 3.78:1.

Glasgow coma scale and score and precipitating factor:

3 patients $(6.98 \%)$ had GCS between 3 and 4, 3 patients $(6.98 \%)$ GCS between 13 and 15.37 $(86.04 \%)$ patients had GCS between 5 and 12 and 
the most important precipitating factor was hypertension (58.14\%) and diabetes (2.33\%).

Location and volume and ventricular extension of hematoma:

40 patients $(93.02 \%)$ had hematoma above the tentorium and 3 patients $(6.98 \%)$ had hematoma below the tentorium. Among the supratentorial hematoma, 4 patients (10\%) had hematoma volume $<30 \mathrm{cc}$ and 36 patients $(90 \%)$ patients had hematoma volume $\geq 30$ cc. 19 patients $(44.19 \%)$ had ventricular extension and 24 patients $(55.81 \%)$ did not have ventricular extension.

\section{Surgical outcome}

Outcome in relation to age

12 patients had favorable outcome and 28 patients had unfavorable outcome who had age $<80$ years. All 3 patients of $\geq 80$ years age had unfavorable outcome and tended towards not significant $(\mathrm{p}=0.26)$.

Outcome in relation to Glasgow coma scale and score

3 patients of GCS 3-4 had unfavorable outcome. In GCS 5-12 group 9 patients had favorable outcome and 28 patients had unfavorable outcome. In GCS 13-15 group all the 3 patients had favorable outcome $(\mathrm{p}=.01)$.

Outcome in relation to location, volume and ventricular extension of hematoma

Among 43 patients 40 patients had supratentorial hematoma. 36 patients had volume of hematoma $\geq 30 \mathrm{cc}$ and 09 patients had favorable outcome. Four patients had volume of hematoma $<30 \mathrm{cc}$, among them 3 patients had favorable outcome $(p=.05)$, which is statistically significant. Among 40 supratentorial hematoma, 12 had favorable outcome and 28 had unfavorable outcome. All 3 patients of infratentorial hematoma had unfavorable outcome. Nineteen patients who had ventricular extension, 2 patients had favorable outcome and 17 patients had unfavorable outcome which tended to statistically significant $(\mathrm{p}=.01)$.

According to Intracerebral Hemorrhage score (ICH score)

Table II: Distribution according to Intracerebral Hemorrhage score (ICH score)

\begin{tabular}{ccc}
\hline ICH Score & No. of Patients & Percentage \\
\hline 0 & 1 & 2.33 \\
1 & 4 & 9.30 \\
2 & 14 & 32.56 \\
3 & 17 & 39.53 \\
4 & 5 & 11.63 \\
5 & 1 & 2.33 \\
6 & 1 & 2.33 \\
\hline Total & 43 & 100.00 \\
\hline
\end{tabular}

Table II shows out of 43 patients 17(39.53\%) patients had ICH score 3 followed by 14(32.56\%) patients who had ICH score $2,5(11.63 \%)$ patients had ICH score 4 and $4(9.30 \%)$ patients had ICH score 1 .

\section{According to Glasgow Outcome Scale}

Table III: According to Glasgow Outcome Scale

\begin{tabular}{lll}
\hline Glasgow Outcome Scale & No. of Patients & Percentage \\
\hline Favorable Outcome & 12 & 27.91 \\
Unfavorable Outcome & 31 & 72.09 \\
\hline Total & 43 & 100.00 \\
\hline
\end{tabular}

Table III shows that $12(27.91 \%)$ patients had favorable outcome and $31(72.09 \%)$ patients had unfavorable outcome in GOS.

According to risk group in relation to Glasgow outcome scale

Table IV: According to risk group in relation to Glasgow outcome scale

\begin{tabular}{lccc}
\hline ICH Score & $\begin{array}{c}\text { Favorable } \\
\text { Outcome }\end{array}$ & $\begin{array}{c}\text { Unfavorable } \\
\text { Outcome }\end{array}$ & p value \\
\hline $\begin{array}{l}\text { Lower risk group } \\
\text { (ICH Score:0-1) }\end{array}$ & 4 & 1 & 0.0168 \\
$\begin{array}{l}\text { Higher risk group } \\
\text { (ICH Score:2-6) }\end{array}$ & 8 & 30 & \\
\hline
\end{tabular}

Table IV shows the risk stratification in relation to GOS was found statistically significant $(\mathrm{p}=.0168)$ by Fisher's exact test. Out of 43 patients 5 patients was in lower risk group in whom 4 patients had favorable outcome and 1 patient had unfavorable outcome. In higher risk group out of 38 patients 8 patients had favorable outcome and 30 patients had unfavorable outcome.

Table V: Correlation between ICH score with Glasgow Outcome Scale

\begin{tabular}{llll}
\hline $\begin{array}{l}\text { Independent } \\
\text { variable }\end{array}$ & $\begin{array}{l}\text { Dependable } \\
\text { Variable }\end{array}$ & $\begin{array}{l}\text { Correlation } \\
\text { Coefficient (r) }\end{array}$ & p-value \\
\hline ICH score & GOS & -0.635 & 0.001 \\
\hline
\end{tabular}

Table V shows the correlation between ICH score with GOS was done and found statistically highly significant $(\mathrm{p}=.001)$. Negative correlation between ICH score with GOS was found.

\section{Discussion}

Spontaneous intracerebral hemorrhage (ICH) comprises $10-15 \%$ of all strokes and is associated with significant morbidity and mortality. Survivors often face lifelong disability and management of $\mathrm{ICH}$ remains baffling and proven treatments for $\mathrm{ICH}$, whether medical or surgical, do not exist, and all clinical trials to date have failed to show effective treatment strategies ${ }^{\mathbf{6 - 9}}$.

Clinical grading scales provide a framework for decision-making and efficacy assessment for novel 
therapeutics. The ICH score is commonly used in the setting of ICH to stratify patients for their risk of poor outcome. Despite being derived to predict 30-day mortality, the ICH score can be valuable in predicting functional disability or functional outcome $\mathbf{8}^{\mathbf{8}}$. Previous studies have shown the ability of the ICH score to predict functional outcome as measured by $\mathrm{mRS}$ at 12 months ${ }^{10}$ and disability as measured by GOS at six months. ${ }^{11}$ In our study we have tried to see the mortality and functional outcome and/or disability in terms of GOS in a very short period of 30 days.

We found co-morbidities like hypertension a very important predisposing factor $(58.14 \%)$ for spontaneous intracerebral hemorrhage. Diabetes and hypertension together were present in $8(18.60 \%)$ patients. All together $77 \%$ of patients had hypertension in our series which is almost similar to the findings of $73 \%$ of hypertensive patients in the series of Hemphill et $\mathrm{al}^{5}$.

In the present study the mean \pm SD age of the study population was $57.95 \pm 12.38$ years ranging from 28 to 82 years and $6.89 \%$ of patients were $\geq 80$ years of age. Hemphill et al. ${ }^{\mathbf{5}}$ in their study found mean age to be $66 \pm 15$ years ranging 22 to 91 years and $22 \%$ were of age $\geq 80$ years. Age above or below 80 years was not significant in relationship to surgical outcome. Possible reason was that number of patients aged $\geq 80$ years was small and they presented with low GCS. We had male predominance (Male $79 \%$ and Female 20.93\%) in our study.

As GCS score is the maximally associated factor in relation to outcome, it is given the most importance in the scale. The GCS score is divided into 3 subgroups (GCS scores of 3 to 4, 5 to 12, and 13 to $15)$ to reflect the very strong influence of GCS on outcome accurately. Hemphill et al. ${ }^{5}$ found that 1 of $35(2.9 \%)$ patient with a presenting GCS scores of 3 or 4 survived to 30 days, and only 5 of $60(8.3 \%)$ patients with a presenting GCS scores of 13 to 15 died, whereas 29 of $57(50.8 \%)$ patients with a GCS score of 5 to 12 died within 30 days. In our study 3 patients presented with GCS score of 3 or 4 and all 3 of them $(100 \%)$ died. Of the 37 patients with a GCS score of 5 to $12,9(24.32 \%)$ patients had favorable outcome and all the 3 patients (100 $\%$ ) with a GCS score of 13 to 15 had favorable outcome. Glasgow Coma Scale was found to have significant relationship $(\mathrm{p}=0.01)$ with the surgical outcome.

Volume of hematoma is another very important factor in management and outcome prediction of intracerebral hemorrhage patient. In this study the volume of hematoma ranged from 21 to $150 \mathrm{cc}$ $(55.08 \pm 29.96)$. $4(10 \%)$ of the patients with supratentorial hematoma had hematoma volume of $<30 \mathrm{cc}$ whereas $36(90 \%)$ patients had hematoma volume of $\geq 30 \mathrm{cc}$. Volume of the hematoma was found to have significant $(p=0.05)$ relationship with the surgical outcome of spontaneous intracerebral hematoma, the greater the hematoma volume the worse the outcome, which is similar to the findings in other studies $(p=0.047)^{\mathbf{5}}$. Broderick et al. ${ }^{\mathbf{1 2}}$ found patients with a parenchymal hemorrhage volume of $60 \mathrm{~cm}^{3}$ or more on their initial computed tomogram and a GCS score of 8 or less had a predicted 30-day mortality of $91 \%$. Patients with a volume of less than $30 \mathrm{~cm}^{3}$ and a GCS score of 9 or more had a predicted 30-day mortality of $19 \%$. This finding was $96 \%$ sensitive and $98 \%$ specific. This indicates that the GCS and volume of hematoma together are very important predictors in outcome.

Location of the hematoma in relation to tentorium can also provide good prediction of outcome in cases of ICH. In this study $40(93.02 \%)$ patients had supratentorial and $3(6.98 \%)$ had infratentorial hematoma. We did not find location of the hematoma to be a significant one $(p=0.02)$ which was similar to the findings of the study by Broderick $^{12}$.

Intracerebral hemorrhage with ventricular extension had a higher mortality $(66 \%)$ compared to the patients without ventricular extension (19\%) in the study by Hemphill et al. ${ }^{\mathbf{5}}$. In the present study 19(44.19\%) patients had ventricular extension and $10(52.63 \%)$ of them died whereas $24(55.81 \%)$ patients had no ventricular extension and $5(20.83 \%)$ of them died, which shows that there is significant $(\mathrm{p}=0.01)$ relationship between the surgical outcome and ventricular extension of $\mathrm{ICH}$.

Regarding outcome $78 \%$ of patient with ICH score $1,82 \%$ with ICH score 2 and $100 \%$ with ICH score $3,4,5$ had modified Rankin Score of $\geq 4$ at discharge ${ }^{13}$. At 6 months after ICH $34.6 \%$ were dead, 38.6\% had good outcome (GOS 4 to 5) and $22.8 \%$ were living with significant impairment $(\text { GOS } 2 \text { to } 3)^{\mathbf{1 4}}$. In this study 12 patients had favorable outcome and 31 patients had unfavorable outcome. One (100\%) patient of ICH score 0 and 3 $(75 \%)$ out of 4 patients of ICH score 1, were independent on their $30^{\text {th }}$ post ictus day. Fernandes et al. ${ }^{15}$ in their study found that $70 \%$ patient of $\mathrm{ICH}$ score 0 and $30 \%$ of ICH score 1 were independent at the time of discharge ranging from $2-4$ weeks. We feel that the better outcome in our study in patients of ICH score of 0 and 1 was beacause of our smaller sample size than Fernandes'. On the other side, 7(50\%) of our patients with ICH score 2 and $1(2.8 \%)$ patient with ICH score 3 were independent on their $30^{\text {th }}$ post ictus day, whereas 
$2 \%$ of patients of ICH score 2 and 5\% of ICH score 3 were independent at the time of discharge in the series of Fernandes et al. ${ }^{15}$. In this study in the patients with ICH score 2, better outcome was found. From the findings of our study we can come to the inference to include ICH score 2 patients in the lower risk group as they had significant $(50 \%)$ better outcome. Risk stratification in relation to GOS was also found statistically significant $(\mathrm{P}=$ $0.0168)$.

Different studies have found 30 days mortality rates for ICH score of $1,2,3$, and 4 to be ranging from $2.9 \%-13 \%, 22 \%-30.8 \%, 61.1 \%-75 \%$ and $68 \%$ $97 \%$ respectively. In these studies it was evident that each increase in the ICH Score was associated with a progressive increase in 30-day mortality, ${ }^{5}, 13$ ${ }^{15}$. In this study the relation between ICH score and surgical outcome of spontaneous intracerebral hematoma was found highly significant $(\mathrm{p}=0.001)$ and they had inverse relation with $r$ value of 0.635 in Pearson's correlation coefficient test. It is well established from this and previous other studies that the lower the ICH score the better the outcome or the vice versa. Mortality rates became high with a very low rate of good outcome when the ICH score was $\geq 3^{11}$.

Conclusions: The ICH score is a simple clinical grading scale that allows risk stratification of patients with intracerebral hemorrhage. As correlation between ICH score and surgical outcome of spontaneous intracerebral hematoma was found statistically very significant, it recommend its widespread use as a grading scale for preoperative counseling. The use of ICH score can improve standardization of clinical treatment protocols for ICH.

Recommendations: As this was a study in small group of population with short follow-up period, we recommend large number of populations and multicenter studies with longer follow-up period for better assessment of post-operative patient status in ICH. As ICH score correlates the surgical outcome, we also recommend that preoperative ICH score assessment is necessary in every center.

\section{Reference:}

1. Aghi M, Ogilvy CS \& Carter BS.Surgical Management of Intracerebral Hemorrhge. In HH Schmidek \& DW Roberts (eds), Operative Neurosurgical Techniques: Indications, Methods, Results, 5th edn, Saunden Elsevier, Edinburg, 2006; 1062-1074.

2. Andaluz $\mathrm{N}$ and Zuccarello $\mathrm{M}$. Recent trends in the treatment of spontaneous intracerebral hemorrhage: analysis of a nationwide inpatient database. J Neurosurg 2009; 110:403-410.
3. Brott T, Adams HP, Olinger CP, Marler JR, Barsan WG, Biller J, Spilker J, Holleran R, Eberle R \& Hertzberg V. Measurements of acute cerebral infarction: a clinical examination scale. Stroke 1989; 20: 864-870.

4. Spetzler RF and Martin NA. A proposed grading system for arteriovenous malformations. J Neurosurg 1986; 65: pp. 47.

5. Hemphill JC, Bonovich DC, Besmertis L \& Manley GT. ICH Score: A simple, reliable grading scale for intracerebral hemorrhage. Stroke 2001; 32: 891-897.

6. Shokouhi G, Farhoudi M, Afrough A and Hamdi A. Prediction of Intracerebral Hemorrhage Outcome. Res. J Biol. Sci 2009; 4(1): 7-10.

7. Caplan LR. Intracerebral Haemorrhage. Lancet 1992; 339: 656-658.

8. Appelboom G, Bruce SS, Han J, Piazza M, Hwang B, Hickman ZL, Zacharia BE, Carpenter A, Monahan AS and Vaughan K, Badjatia N and Connolly. Functional outcome prediction following intracerebral hemorrhage. Journal of Clinical Neuroscience 2012; 19: 795-798.

9. Broderick J, Connolly S, Feldmann E, et al. Guidelines for the management of spontaneous intracerebral hemorrhage in adults: 2007 update: a guideline from the American Heart Association/American Stroke Association Stroke Council, High Blood Pressure Research Council, and the Quality of Care and Outcomes in Research Interdisciplinary Working Group. Stroke 2007;38:2001-23.

10. Hemphill JC, Farrant M, Neill Jr TA. Prospective validation of the ICH Score for 12-month functional outcome. Neurology 2009; 73:1088-94.

11. Godoy DA, Pinero G \& Napoli M. 'Predicting mortality in spontaneous intracerebral hemorrhage: can modification to original score improve the prediction? Stroke 2006; 37: 1038-1044.

12. Broderick JP, Brott TG, Duldner JE, Tomsick T and Huster G. Volume of intracerebral hemorrhage. A powerful and easy-to-use predictor of 30-day mortality. Stroke 1993;24:987-993.

13. Jamora RDG, Kishi-Generao EM \& Bitanga ES. The ICH Score: Predicting mortality and functional outcome in an Asian population. Stroke 2003;34:6-7.

14. Godoy DA \& Boccio A. ICH score in a rural village in the Republic of Argentina. Stroke 2003; 34: 150-151.

15. Fernandes H, Gregson BA, Siddique MS, Mendelow AD, Hemphill, Bonovich DC, Johnston SC \& Manley GT. Testing the ICH score: response. Stroke 2002; 33: $1455-1456$ 